\title{
ELISA para la Identificación de los Patrones Alimentarios de Triatominae en Colombia
}

\author{
Using ELISA for identifying Triatominae feeding behaviour in \\ Colombia
}

Ana E. Farfán ${ }^{1}$, Reinaldo Gutiérrez ${ }^{2}$ y Víctor M. Angulo ${ }^{1}$

1 Centro de Investigaciones en Enfermedades Tropicales. CINTROP. Universidad Industrial

de Santander. elvirafarfan01@hotmail.com, pitorio@hotmail.com

2 Universidad de Pamplona. regumar@unipamplona.edu.co,

Recibido 22 Enero 2007/Enviado para Modificación 29 Julio 2007/Aceptado 25 Septiembre 2007

\section{RESUMEN}

Objetivo Estandarizar la técnica de ELISA para la identificación de los patrones alimentarios de insectos triatominos.

Métodos Para estandarizar el ELISA se prepararon 12 antisueros policlonales anti-especie animal a partir de inmunizaciones en conejos con sueros de animales domésticos y silvestres, que fueron titulados y absorbidos para mejorar especificidad; como controles positivos se utilizaron contenidos intestinales de ninfas de $\mathrm{V}$ instar de Rhodnius prolixus previamente alimentadas sobre cada hospedero; los negativos se obtuvieron de triatominos sin alimentar. Se procesaron 60 contenidos intestinales de $R$. prolixus de intradomicilio colectados en campo para determinar la efectividad de la prueba.

Resultados Se estandarizó la técnica de ELISA con alta reactividad en la detección de cada proteína sanguínea de los controles positivos utilizados. En el $70 \%$ de los contenidos intestinales de los triatominos colectados en el domicilio se detectaron proteínas sanguíneas de uno o más hospederos domésticos y silvestres. Sangre de ave, humano, cerdo y cabra fueron las fuentes alimentarias más frecuentes; proteínas sanguíneas de animales silvestres fueron identificadas en $9,5 \%$.

Conclusiones La técnica mostró ser efectiva en la detección de proteínas sanguíneas de diferentes hospederos en el contenido intestinal de triatominos de laboratorio y de campo. Si bien se determinó preferencialmente sangre de animales domésticos, el hallazgo de sangre de animales silvestres podría indicar movilidad de insectos probablemente de origen silvestre al domicilio. Esta herramienta ayuda a entender el comportamiento de los triatominos frente a sus hospederos, contribuyendo a la comprensión de la ecoepidemiología de la Enfermedad de Chagas.

Palabras Clave: Triatominae, ELISA, patrón alimentario (fuente: DeCS, BIREME). 


\section{ABSTRACT}

Objective Standardising the ELISA technique for identifying triatomine insects' feeding behaviour.

Methods The ELISA test was standardised by preparing 12 animal anti-specie polyclonal antisera by immunising rabbits with sera from domestic and wild animals; the sera were titred and absorbed to improve specificity. The intestinal content of fifth-instar Rhodnius prolixus (previously fed on each host) was used as positive control; negative controls were obtained from triatomines without feeding. The intestinal content from 60 intradomicile $R$. prolixus collected in the field was processed to determine the test's effectiveness.

Results The high-reactivity ELISA technique was standardised in detecting every blood protein in the positive controls used here. Blood proteins from one or more domestic and wild hosts were detected in $70 \%$ of the intestinal content of triatomines collected in homes. Bird, human, pig and goat blood were the most frequent feeding sources; blood proteins from wild animals were identified in $9.5 \%$ of them.

Conclusions The technique was shown to be effective in detecting blood proteins from different hosts in the intestinal content of triatomines taken from the laboratory and the field. Even though domestic animals' blood was preferentially determined, the findings from wild animals' blood could indicate insect mobility probably from the wild to the domicile. This tool helps in understanding triatomines' behaviour regarding their hosts, thereby contributing to understanding Chagas' disease ecoepidemiology.

Key Words: Triatominae, ELISA, feeding behaviour (source: MeSH, NLM).

L a Enfermedad de Chagas producida por Trypanosoma cruzi afecta aproximadamente a 17 millones de personas en América y es transmitido por diferentes especies de triatominos que tienen hábitats intradomiciliarios y extradomicilarios (1). Una de las estrategias para conocer el comportamiento de estos vectores respecto al hábitat humano, es la identificación de sus patrones alimentarios, que contribuye al conocimiento de su dinámica de dispersión y potencial adaptación a diferentes hábitats y hospederos (2).

La necesidad de implementar estrategias de control y vigilancia efectivos en especies que ocupan hábitats intradomiciliarios y de especies que incursionan a éstos desde ecótopos silvestres próximos, obliga a conocer aspectos de su biología y eco-epidemiología. La ausencia de estudios en Colombia sobre el comportamiento alimentario de los triatominos de hábitats relacionados con la actividad humana y responsables de la transmisión de T. cruzi en las zonas 
REVISTADE SALUD PÚBLICA• Volumen 9 (4), Diciembre 2007

endémicas, motivó la estandarización de la técnica de ELISA, utilizando material entomológico procedente de laboratorio y de campo.

Desde los años 60, se ha intensificado la búsqueda de métodos rápidos, prácticos, sensibles y específicos que brinden datos confiables sobre las fuentes sanguíneas utilizadas por los insectos. Las pruebas inmunológicas más utilizadas como el test de precipitinas, la inmunodifusión doble y las reacciones de hemaglutinación, presentan baja sensibilidad, necesitan gran cantidad de material biológico y la interpretación de sus resultados es bastante subjetiva $(3,4)$. Recientemente, las pruebas moleculares (5) y el inmunoensayo enzimático "Enzyme-Linked Immunosorbent Assay" (ELISA) (6), adaptado para estos estudios en 1981 (7), han mostrado alta sensibilidad, especificidad y confiabilidad comparadas principalmente con el test de precipitinas $(3,4)$.

\section{MATERIALES Y METODOS}

Antisueros policlonales fueron producidos en conejos de raza Nueva Zelanda, utilizando como antígenos para la inmunización sueros (proteínas totales) de humano, animales domésticos (ave, bovino, cabra, canino, equino, gato, ovino y porcino) y silvestres (armadillo (Dasypus novemcinctus), rata (Rattus rattus) y opossum (Didelphis marsupialis)) (8). Estos antisueros fueron titulados mediante la técnica de ELISA adaptada para este estudio (3,6,7); los títulos fueron definidos por el recíproco de la dilución más alta del suero en el que se observó reacción con su homólogo. Se determinó reactividad cruzada, tomando como punto de corte la media de las absorbancias más dos desviaciones estándar. Para reducir la concentración de anticuerpos heterólogos y mejorar su especificidad, los antisueros fueron absorbidos mediante precipitación en medio líquido con modificaciones al protocolo de Weitz (9); ésto se repitió hasta que los valores de absorbancia no superaran el punto de corte; titulaciones y reacciones cruzadas adicionales fueron realizadas. Para determinar la reactividad de estos antisueros en la detección de proteínas sanguíneas se disectaron triatominos alimentados por xenodiagnóstico natural y sin alimentar (controles positivos y negativos). Estos controles en diferentes diluciones reaccionaron con los antisueros heterólogos y homólogos previamente diluidos y con éstos se estableció un punto de corte.

Se incluyeron 60 ejemplares de $R$. prolixus (9 ninfas y 51 adultos) que fueron colectados por búsqueda activa en el intradomicilio en el período de Mayo de 2003 a Enero del 2006 en 35 viviendas de áreas rurales de los departamentos de Arauca, Casanare y Santander; éstos fueron identificados 
por medio de la clave de Lent \& Wygodzinsky (10). Sus contenidos intestinales fueron removidos y almacenados a $-20^{\circ} \mathrm{C}$ y posteriormente procesados en el ELISA.

Los patrones alimentarios de $R$. prolixus fueron determinados por la reactividad con el antisuero específico; se estableció la proporción de hospederos identificados.

Aspectos éticos

En el manejo de animales se siguieron las normas éticas según la Ley 84 de 1989 y la Resolución No. 008430 de 1993 del Ministerio de Protección Social de Colombia.

\section{RESULTADOS}

Las máximas diluciones reactivas de los doce antisueros se establecieron en un rango de 1/128 000 hasta 1/512 000; los mismos rangos se observaron en los títulos de conejos inmunizados pareados y en los duplicados de las titulaciones. Los antisueros que presentaron el menor título fueron ave y ovino (1/128 000) y los más altos correspondieron a cabra, armadillo, opossum y gato. Las reacciones inespecíficas detectadas mediante el ELISA en todos antisueros heterólogos no absorbidos, excepto para ave, fueron eliminadas con dos absorciones aplicadas; una disminución en los títulos, que varió desde 1/800 hasta 1/51 200 fue observada. En el ELISA utilizando los controles positivos, se observó reactividad específica de los antisueros, con valores de absorbancia que oscilaron desde 0,15 hasta 2,7 , superiores al punto de corte preestablecido (Figura 1).

Figura 1. Reactividad obtenida en el ELISA de contenidos intestinales de R. prolixus

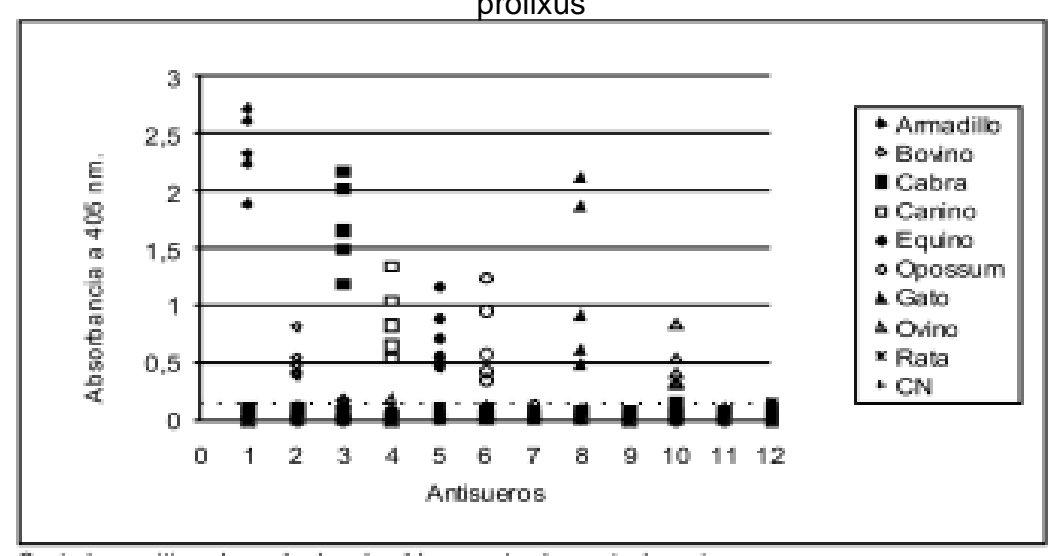

Cantroks positivos: Ieyenda derecha. Linea pu nteadac punto de corte. 
REVISTADE SALUD PÚBLICA· Volumen 9 (4), Diciembre 2007

De los 60 contenidos intestinales de R. prolixus de campo analizados, 42 fueron positivos (70 \%) para uno o más hospederos. Los hospederos domésticos fueron identificados en mayor proporción (90,5 \%) y en entre éstos las aves mostraron un alto porcentaje (85,7), seguido de humano, porcino y caprino (Tabla 1). En Arauca y Casanare se detectó sangre de animales silvestres como armadillo, opossum y rata en 9,5 \% (4/42) (Tabla 1). En 12 (28,6 \%) ejemplares fueron identificadas múltiples alimentaciones, de los cuales $50 \%$ correspondieron a dos hospederos; en los insectos con tres y cuatro fuentes ( 25 y 16,7 \% respectivamente) se destacó la frecuente asociación con ave. En $45 \%$ de los contenidos intestinales de R. prolixus se observó un grado de digestión alto (poco contenido intestinal) y en 18 de éstos la prueba de ELISA logró identificar proteínas sanguíneas.

Tabla 1. Hospederos de R. prolixus colectados en intradomicilio de áreas rurales identificados mediante la técnica de ELISA ${ }^{a}$

\begin{tabular}{|c|c|c|c|c|c|c|c|c|}
\hline \multirow{3}{*}{ Hospederos } & \multicolumn{6}{|c|}{ Departamentos } & \multirow{2}{*}{\multicolumn{2}{|c|}{ Total }} \\
\hline & \multicolumn{2}{|c|}{ Arauca } & \multicolumn{2}{|c|}{ Casanare } & \multicolumn{2}{|c|}{ Santander } & & \\
\hline & $n$ & $\%$ & $n$ & $\%$ & $\mathrm{n}$ & $\%$ & $n$ & $\%$ \\
\hline Armadillo & 1 & 20 & 1 & 5,6 & 0 & 0 & 2 & 4,8 \\
\hline Opossum & 0 & 0 & 1 & 5,6 & 0 & 0 & 1 & 2,4 \\
\hline Rata & 0 & 0 & 1 & 5,6 & 0 & 0 & 1 & 2,4 \\
\hline Humano & 0 & 0 & 5 & 27,8 & 2 & 10,5 & 7 & 16,7 \\
\hline Canino & 0 & 0 & 3 & 16,7 & 0 & 0 & 3 & 7,1 \\
\hline Equino & 0 & 0 & 1 & 5.6 & 1 & 5,3 & 2 & 4,8 \\
\hline Bovino & 0 & 0 & 0 & 0 & 0 & 0 & 0 & 0 \\
\hline Ave & 5 & 100 & 13 & 72,2 & 18 & 94,7 & 36 & 85,7 \\
\hline Gato & 0 & 0 & 1 & 5,6 & 2 & 10,5 & 3 & 7,1 \\
\hline Ovino & 0 & 0 & 1 & 5.6 & 0 & 0 & 1 & 2,4 \\
\hline Porcino & 0 & 0 & 4 & 22,2 & 3 & 15,8 & 7 & 16,7 \\
\hline Caprino & 0 & 0 & 3 & 16,7 & 3 & 15,8 & 6 & 14,3 \\
\hline Positivos & 5 & 71,4 & 18 & 52,9 & 19 & 100 & 42 & \\
\hline Negativos & 2 & 28,6 & 16 & 47,1 & 0 & 0 & 18 & \\
\hline
\end{tabular}

las alimentaciones múltiples en algunos insectos.

\section{DISCUSION}

La identificación de los hospederos de insectos hematófagos permite aproximarse al conocimiento de su comportamiento respecto al hombre y a su hábitat. La búsqueda de métodos para su identificación ha posicionado a las pruebas serológicas como una buena opción y ELISA ha demostrado ser una herramienta altamente sensible y específica $(3,4,6)$. En este trabajo, se logró la producción de anticuerpos policlonales contra proteínas totales de sangre de animales domésticos, silvestres y humanos con altos títulos, similares a los mostrados por otros autores $(3,7)$. La reactividad cruzada entre las diferentes especies de animales, especialmente en los mamíferos fue eliminada como en otros trabajos, con la absorción de anticuerpos heterólogos, con mejoría en la especificidad (3,7). A 
pesar de la apreciable disminución en los títulos de los antisueros de animales muy próximos en la escala zoológica como caprino, bovino y ovino, observado en este estudio y reportada por otro autor (9), en la prueba de ELISA se detectó reactividad específica ante sus homólogos, con títulos por encima del punto de corte. En Colombia, es limitada la producción de estos antisueros y comercialmente solo se puede acceder a algunos de ellos; su producción en laboratorios de investigación o de referencia, permitirá su amplia utilización en el análisis de los patrones alimentarios de especies de triatominos y otros insectos como herramienta útil en estudios biológicos y epidemiológicos.

La eficacia de la identificación de los patrones alimentarios de los triatominos depende de varios factores como el volumen de ingesta y el grado de digestión de las proteínas ingeridas, y ésta a su vez del tiempo transcurrido desde su alimentación hasta su disección en el laboratorio (9); en este trabajo el ELISA en contenidos intestinales de $R$. prolixus con alto grado de digestión, logró identificar trazas de proteínas sanguíneas; además un mínimo volumen (menor a 10 ul) permitió el análisis del contenido intestinal de un insecto frente a doce antisueros. El uso de esta estrategia para conocer el comportamiento de los insectos debe estar relacionado con las características de sus hábitats, lugares de colecta, conocimiento del tipo y densidad de los reservorios de la zona, pues permite un mejor análisis e interpretación de los resultados.

El análisis de los patrones alimentarios de $R$. prolixus colectados en campo, permitió conocer el carácter eurófago que parece predominar en su alimentación y su preferencia alimentaria por animales domésticos, ésto puede ser debido a la disponibilidad de esos animales en las zonas, constituyendo un factor preponderante para la selección alimentaria y factor de riesgo para su domiciliación. Humano se identificó en menor proporción, comparado con un trabajo de Venezuela en donde proteínas sanguíneas humanas se identificaron en 58,3 \% (11). El hallazgo de hospederos silvestres en los contenidos intestinales de $R$. prolixus colectados en intradomicilio de los departamentos de Arauca y Casanare podría indicar, la posible incursión de los insectos desde hábitats silvestres hacia el domicilio, lo que constituye en factor de riesgo para la transmisión de T. cruzi al hombre en estas zonas

Agradecimientos. A las Bacteriólogas Laura Rodriguez y Yenny Castellanos, por su colaboración en la preparación de algunos antisueros, al personal del laboratorio de entomología del CINTROP y a las comunidades de los departamentos muestreados. Financiación: COLCIENCIAS. Proyectos códigos No. 11020413029 y 11020413010.

Conflictos de intereses: Ninguno 
REVISTADE SALUD PÚBLICA · Volumen 9(4), Diciembre 2007

\section{REFERENCIAS}

1. Moncayo A. The global epidemiology of infectious diseases. Chagas disease. World Health Organization 2004. Capítulo 11:325-348 [Internet]. Disponible en: http:// whqlibdoc.who.int/publications/2004/9241592303_chap11.pdf Consultado: Octubre de 2006.

2. Zeledón R, Calvo N, Montenegro VM, Lorosa ES, Arévalo C. A survey on Triatoma dimidiata in an urban area of the Province of Heredia, Costa Rica. Mem Inst Oswaldo Cruz, Rio de Janeiro 2005;100(6):607-612.

3. Duarte R. Ensaio imunoenzimático ELISA para identificação experimental de fontes alimentares em Panstrongylus megistus (Burmeister 1835) (Hemiptera: Reduviidae).Tese de mestrado em Biologia Celular e Molecular IOC / Fundação Oswaldo Cruz, 1997;103 pp.

4. Gomes LAM; Duarte R, Lima DC, Diniz BS, Serrao ML, Labarthe N. Comparison between precipitin and ELISA tests in the bloodmeal detection of Aedes aegypti (Linnaeus) and Aedes fluviatilis (Lutz) mosquitoes experimentally fed on feline, canine and human hosts. Mem Inst Oswaldo Cruz, Rio de Janeiro 2001;96(5):693695.

5. Bosseno MF, Garcia LS, Baunaure F, Gastelum EM, Gutierrez MS, Kasten FL, et al. Identification in triatomine vectors of feeding sources and Trypanosoma cruzi variants by heteroduplex assay and a multiplex miniexon polymerase chain reaction. Am J Trop Med Hyg 2006;74(2):303-305.

6. Voller A, Bartlett A, Bidwell DE. Enzyme immunoassays for parasitic diseases. Trans Roy Soc Trop Med and Hyg 1976;70(2):98-106.

7. Burkot TR, Goodman WG, DeFoliart GR. Identification of mosquito blood meals by Enzyme-Linked Immunosorbent Assay. Am J Trop Med Hyg 1981;30(6):13361341.

8. Johnstone A, Thorpe R. Immunochemistry in practice. In: Production of antibodies. Second edition, London, Blackwell scientific publications, 1987;31-34.

9. Weitz B. Identification of blood meals of blood-sucking arthropods. Bull World Health Organ 1956;15:473-90.

10. Lent H, Wygodzinsky P. Revision of the Triatominae (Hemiptera, Reduviidae) and their significance as vector of Chagas disease. Bull Am Mus Nat Hist 1979;163:127-250.

11. Feliciangeli MD, Carrasco H, Patterson JS, Suárez B, Martínez C, Medina M. Mixed domestic infestation by Rhodnius prolixus Stal, 1859 and Panstrongylus geniculatus Latreille, 1811, vector incrimination, and seroprevalence for Trypanosoma cruzi among inhabitants in El Guamito, Lara State, Venezuela. Am J Trop Med Hyg 2004;71(4):501-505. 